\title{
Alimentos e bebidas consumidos por estudantes de odontologia: subsídios para a construção de estratégias de promoção à alimentação saudável
}

\author{
Food and beverages consumed by dentistry students: inputs for \\ creating healthy eating promotion strategies
}

\author{
Deison Alencar Lucietto, ${ }^{1}$ Mayla Mathias Prass, ${ }^{1}$ Ediane Casani, ${ }^{1}$ Sidimar Meira Sagaz \\ 'Faculdade Especializada na Área de Saúde do Rio Grande do Sul, Passo Fundo, RS, Brasil.
}

Recebido em: 23/07/2016 / Aceito em: 27/08/2016 / Publicado em: 30/09/2016

deisonlucietto@hotmail.com

\section{RESUMO}

Introdução e objetivo: a alimentação é fundamental para a construção de modos de vida saudáveis. Evidências científicas mostram a associação entre o maior consumo de alimentos ricos em gordura, sal e açúcar e o incremento de doenças não transmissíveis. Considerando que hábitos alimentares saudáveis devem ser fomentados em espaços como a família e as instituições de ensino, este estudo teve como objetivo descrever os alimentos e as bebidas consumidos por estudantes de Odontologia, durante a permanência nas salas de aula. Método: tratou-se de pesquisa observacional transversal, nas dependências de um Curso de Odontologia no Rio Grande do Sul. Os dados foram coletados de modo indireto através da observação e quantificação de itens descartados nas lixeiras das salas de aula, durante o período de um mês, no ano de 2015. Resultados: ao total, foram contabilizados 1301 itens. Entre os alimentos, identificou-se maior consumo de doces e miscelâneas $(66,7 \%)$, seguido por biscoitos, bolos e cereais $(26,5 \%)$, frutas $(5,2 \%)$ e iogurtes $(1,6 \%)$. Quanto às bebidas, constatou-se maior consumo de café $(52,0 \%)$, seguido por refrigerante $(16,3 \%)$, água $(12,6 \%)$, bebida achocolatada $(8,3 \%)$, suco $(7,1 \%)$ e outros $(3,7 \%)$. Evidenciou-se maior presença de itens ultraprocessados, hipercalóricos, pouco nutritivos e com grande quantidade de açúcares livres. Considerações finais: a realização de atividades de educação em saúde, incentivo à oferta e à comercialização de alimentos e bebidas saudáveis deve ser estimulada como um meio para a promoção da saúde. Instiga-se a adaptação e o uso de métodos alternativos para a verificação do consumo de alimentos e bebidas por grupos de indivíduos, como os descritos neste estudo, em novas investigações.

Palavras-chave: Promoção da saúde; Alimentação; Açúcares livres; Doenças Crônicas; Estudantes de Odontologia.

\section{ABSTRACT}

Introduction and objective: eating is fundamental for creating a healthy lifestyle. Scientific evidence confirm the association of a higher intake of fatty, salty and sugary foods and an increase in non- transmissible diseases. Considering that healthy eating habits must be promoted in spaces such as family and educational institutions, this study aimed at describing the food and beverages consumed by Dentistry students during their time in the classroom. Method: the transversal observational research took place at a Dentistry graduation course in Rio Grande do Sul. Data was collected in an indirect way, through the observation and counting of the items discarded in the classrooms' trash bins during a month, in 2015. Results: in total, 1301 items were counted. Among different types of food, we identified a higher intake of assorted sweets $(66,7 \%)$, followed by cookies, cakes and cereal (26,5\%), fruit (5,2\%) and yogurts (1,6\%). As for drinks, we observed a higher intake of coffee $(52,0 \%)$, followed by soft drinks (16,3\%), water $(12,6 \%)$, chocolate milk beverages (8,3\%), juice (7,1\%) and others (3,7\%). The study showed a higher presence of ultra-processed, hypercaloric, low nutrient items with a high level of free sugar. Closing remarks: the execution of educational activities about health and the support to the offer and selling of healthy food and drinks must be stimulated as a form of promoting health. 
We instigate the adaptation and the use of alternate methods to check the consumption of food and beverages by groups of individuals, such as the one described in this study, in new investigations.

Keywords: Health promotion; Eating; Free sugars; Chronic diseases; Dentistry students.

\section{INTRODUÇ̃̃O}

A construção de sociedades mais saudáveis pressupõe maior controle de indivíduos e comunidades sobre os determinantes da sua saúde. ${ }^{1}$ A alimentação é fundamental para a construção de modos de vida mais saudáveis, ${ }^{2}$ insere-se na cultura e não deve ser reduzida aos aspectos puramente nutricionais. ${ }^{3}$ Evidências científicas confirmam que ela pode trazer, tanto efeitos positivos, quanto negativos sobre a saúde ao longo da vida. ${ }^{4}$ Os hábitos alimentares representam fator de risco comum ${ }^{5}$ a várias doenças crônicas, como cárie dentária, obesidade, diabetes, hipertensão, doenças cardiovasculares, câncer, pedras nas vesículas, nos rins e Doença de Crohn, dentre outras, ${ }^{4,5-12}$ consideradas importantes fontes de morbidade, mortalidade e incapacidade. ${ }^{2,8}$ De acordo com Guia Alimentar da População Brasileira, ${ }^{8}$ os alimentos são classificados em quatro categorias, em função do tipo de processamento empregado na sua produção: 1) in natura ou minimamente processados (obtidos de plantas ou animais, diretamente da natureza, ou submetidos a alterações mínimas); 2) óleos, gorduras, sal e açúcar (para temperar, cozinhar alimentos e criar preparações); 3) processados (produtos fabricados com a adição de sal ou açúcar); e 4) ultraprocessados (que envolvem processamento com a combinação de vários ingredientes, etapas e técnicas). Os alimentos ultraprocessados, frequentemente pobres em fibras, ricos em gorduras, açúcares e sal, interferem negativamente na saciedade, retardando-a ou impedindo que ocorra. ${ }^{8}$

Verifica-se hoje, em nível mundial, maior consumo de alimentos hipercalóricos, pouco nutritivos, com alto teor de gordura, açúcares e sal. ${ }^{8}$ Em função disso, a OMS possui recomendações claras sobre a alimentação: limitar a ingestão de gorduras, açúcares livres e sal e aumentar o consumo de frutas, hortaliças, legumes, cereais integrais e frutas secas. ${ }^{8}$ Ainda, de acordo com o Guia Alimentar da População Brasileira, ${ }^{2}$ os alimentos in natura ou minimamente processados devem ser a base da alimentação. Óleos, gorduras, sal e açúcar devem ser usados em pequenas quantidades. Já, os alimentos processados devem ser limitados e, por fim, o consumo de alimentos ultraprocessados deve ser evitado.

A ingestão elevada de açúcares livres é preocupante, uma vez que se associa à má qualidade do regime alimentar, grande quantidade de energia e sem nutrientes específicos. ${ }^{2,13}$ Sabe-se que ela se relaciona ao preço dos produtos. ${ }^{9,14}$

Os "acúcares livres" dizem respeito aos monossacarídeos e dissacarídeos adicionados a alimentos e bebidas pelo fabricante, pelos cozinheiros ou pelo consumidor e os açúcares naturalmente presentes no mel, xaropes, sucos de frutas e sucos de frutas concentrados. ${ }^{15} \mathrm{O}$ seu consumo elevado está associado a doenças não transmissíveis. ${ }^{13}$ Inclusive, há a hipótese que ele estaria relacionado a mecanismos de depen- dência química, por atuar no sistema de recompensa cerebral. ${ }^{10,11,16,17}$

A recente publicação da diretriz “Ingestão de açúcares por adultos e crianças" ${ }^{13}$ recomenda baixa ingestão de açúcares livres ao longo de toda a vida e a redução da ingestão de açúcares livres a menos de 10\% da ingestão calórica total, ressaltado a importância da medida, tanto em crianças quanto em adultos.

Como as doenças crônicas não transmissíveis são passíveis de prevenção, a partir de mudanças nos padrões de alimentação, ${ }^{2-4,8}$ é preciso promover hábitos alimentares saudáveis em diversos espaços sociais.

A escola, espaço formativo, pode facilitar as escolhas saudáveis, em termos de alimentação. ${ }^{3}$ Tal prerrogativa ficou evidenciada na publicação da Portaria Interministerial $n^{\circ}$. 1010/2006, que instituiu as diretrizes para a promoção da alimentação saudável em escolas públicas e privadas no Brasil.

Existem diferentes métodos disponíveis para verificar a quantidade e qualidade dos alimentos consumidos por indivíduos, dentre eles os diários alimentares, os recordatórios das últimas 24 horas $(\mathrm{R} 24 \mathrm{H})$ e os questionários de frequência alimentar (QFAs). Normalmente estes instrumentos são extensos e/ou exigem respostas dos indivíduos a um grande número de questões.

Estima-se, contudo, que hábitos alimentares de grupos de pessoas (coletivos) possam ser verificados a partir de meios alternativos.

Considerando a relevância de pesquisar sobre hábitos alimentares em nível coletivo, como um meio para planejar ações de promoção da saúde, este estudo teve como objetivo descrever os alimentos e as bebidas consumidos por estudantes de Odontologia, durante a permanência nas salas de aula, de modo indireto, através dos itens descartados nas lixeiras.

\section{MÉTODO}

Tratou-se de pesquisa observacional transversal ${ }^{21}$ nas dependências do Curso de Odontologia da Faculdade Especializada na Área de Saúde do Rio Grande do Sul (FASURGS), na cidade de Passo Fundo/RS. A realização da pesquisa foi autorizada pela Direção e não foi submetida à apreciação do CEP, por tratar de resíduos.

Fizeram parte da amostra todas as salas de aula utilizadas pelos estudantes do primeiro ao último semestre do Curso (em torno de 400 indivíduos). Os dados foram coletados de modo indireto através da observação e quantificação de alimentos e bebidas descartados pelos estudantes nas lixeiras das salas de aula. Foram coletados todos os sacos com o lixo das salas por cinco semanas consecutivas, entre os dias 03/11/2015 e 04/12/2015. Previamente ao início da coleta, foi feito um piloto para planejar a logística e construir os instrumentos de análise.

Para garantir que não houvesse mudança dos hábitos alimentares no período, os estudantes não foram informados. Sacos de lixo devidamente etiquetados foram fornecidos às funcionárias da limpeza, que colaboraram com o recolhimento. Duas vezes por semana, eles foram discretamente recolhidos do armazenamento temporário e levados até uma sala reservada para serem quantificados.

De modo a facilitar a organização dos resíduos foi 
construído um banner em lona lavável com um quadro para depositar os alimentos e as bebidas. Para a quantificação dos resíduos foi construída uma ficha intitulada "Quadro de análise dos alimentos consumidos", onde foram criados grupos para: 1) biscoitos, bolos e cereais; 2) doces e miscelâneas; 3) frutas; 4) iogurtes; e 5) bebidas. Cada um destes grupos contemplava itens específicos. Tanto o banner quanto a ficha foram construídos tomando por base o estudo piloto e dois questionários de frequência alimentar: o QFA-Adultos ${ }^{19}$ e o QFA-Açúcar. ${ }^{20}$

O manuseio dos resíduos foi realizado, utilizando-se equipamentos de proteção individual (avental, gorro, óculos de proteção, máscara e luvas). As embalagens de alimentos e bebidas foram fotografas, incluindo a visão frontal e a informação nutricional. Foi preenchida uma ficha para cada saco de lixo. Após isso, os resíduos foram acondicionados nas suas embalagens originais e descartados no local convencionalmente utilizado para esse fim.

Foi construído um banco de dados no Programa Microsoft Excel, em que foram contabilizados os resultados de cada ficha. A análise dos alimentos e bebidas foi realizada através de procedimentos de estatística descritiva básica, considerando a presença e a porcentagem de cada grupo.

\section{RESULTADOS}

Os resultados são apresentados por grupos e por itens individuais, tomando por base o número de embalagens ou de unidades encontradas. Eles estão expressos em valores absolutos e em percentuais.

Foram analisados resíduos de 53 sacos de lixo, contabilizando, entre alimentos e bebidas, 1301 itens (100\%). Observou-se maior consumo de alimentos $(n=$ 1007) $(77 \%)$ em relação às bebidas $(n=294)(23 \%)$.

\section{Alimentos consumidos}

Conforme a Tabela 1, do total de alimentos consumidos (1007), identificou-se maior consumo dos seguintes grupos: doces e miscelâneas $(672,66,7 \%)$; biscoitos, bolos e cereais $(267,26,5 \%)$; frutas (52, $5,2 \%)$; e iogurtes $(16,1,6 \%)$.

Tabela 1 - Grupos de alimentos consumidos por estudantes de Odontologia nas salas de aula.

\begin{tabular}{lcc}
\hline Alimentos & $\mathbf{N}^{\circ}$ de itens & $\%$ \\
\hline Doces e miscelâneas (por embalagem) & 672 & 66,7 \\
Biscoitos, bolos e cereais (por embalagem) & 267 & 26,5 \\
Frutas (por unidade) & 52 & 5,2 \\
logurtes (por embalagem) & 16 & 1,6 \\
Total alimentos & 1007 & 100,0 \\
\hline
\end{tabular}

Fonte: Dos autores, 2016.

A Tabela 2 traz a especificação dos itens específicos pertencentes a cada grupo de alimentos consumidos pelos estudantes no período da pesquisa.

Quanto ao grupo dos biscoitos, bolos e cereais (por embalagem), constatou-se que os itens de menor consumo foram amendoim, wafer e cachorro quente $(1,0,4 \%)$. Os itens menos consumidos do grupo de doces e miscelâneas (por embalagem) foram pipoca doce e rapadura ( $1,0,1 \%)$. Com relação às frutas (por unidade), a ameixa foi o item de menor presença (1,2\%). Por fim, constatou-se que o iogurte natural foi o item menos consumido $(1,6,2 \%)$ no grupo dos iogurtes (por embalagem).
Tabela 2 - Grupos e itens de alimentos consumidos por estudantes de Odontologia nas salas de aula.

\begin{tabular}{|c|c|c|}
\hline Doces e miscelâneas (por embalagem) & $\mathbf{N}^{\circ}$ de itens & $\%$ \\
\hline Bala individual & 229 & 34,1 \\
\hline Chiclete individual & 149 & 22,2 \\
\hline Bombom/trufa & 115 & 17,1 \\
\hline Chiclete caixinha/envelope & 56 & 8,3 \\
\hline Bala caixinha/envelope & 47 & 7,0 \\
\hline Chocolate barrinha & 27 & 4,0 \\
\hline Pirulito & 24 & 3,7 \\
\hline Confeito & 7 & 1,0 \\
\hline Paçoca & 7 & 1,0 \\
\hline Chocolate barra & 4 & 0,7 \\
\hline Picolé & 3 & 0,4 \\
\hline Goma & 2 & 0,3 \\
\hline Pipoca doce & 1 & 0,1 \\
\hline Rapadura & 1 & 0,1 \\
\hline Total & 672 & 100,0 \\
\hline \multicolumn{3}{|l|}{ Biscoitos, bolos e cereais (por embalagem) } \\
\hline Cereal & 75 & 28,1 \\
\hline Biscoito salgado & 66 & 24,7 \\
\hline Salgadinho & 33 & 12,4 \\
\hline Biscoito doce recheado & 30 & 11,2 \\
\hline Bolinho & 22 & 8,2 \\
\hline Biscoito temperado & 20 & 7,5 \\
\hline Biscoito doce & 18 & 6,7 \\
\hline Amendoim & 1 & 0,4 \\
\hline Wafer & 1 & 0,4 \\
\hline Cachorro quente & 1 & 0,4 \\
\hline Total & 267 & 100,0 \\
\hline \multicolumn{3}{|l|}{ Frutas (por unidade) } \\
\hline Maçã & 25 & 48,1 \\
\hline Banana & 24 & 46,1 \\
\hline Pêssego & 2 & 3,8 \\
\hline Ameixa & 1 & 2,0 \\
\hline Total & 52 & 100,0 \\
\hline \multicolumn{3}{|l|}{ logurtes (por embalagem) } \\
\hline logurte de frutas & 12 & 75,0 \\
\hline logurte light & 2 & 12,6 \\
\hline logurte desnatado & 1 & 6,2 \\
\hline logurte natural & 1 & 6,2 \\
\hline Total & 16 & 100,0 \\
\hline
\end{tabular}

Fonte: Dos autores, 2016

\section{Bebidas consumidas}

Do total de bebidas consumidas (294), identificou-se que houve menor consumo dos grupos energético e chá $(3$, $1 \%$ ), este identificado pelos resíduos do copo (Tabela 3).

Tabela 3 - Grupos de bebidas e itens consumidos por estudantes de Odontologia nas salas de aula.

\begin{tabular}{lcc}
\hline Bebidas (por embalagem) & $\mathbf{N}^{\circ}$ de itens & $\%$ \\
\hline Café & 153 & 52,0 \\
Refrigerante lata & 36 & 12,2 \\
Água sem gás & 29 & 9,9 \\
Bebida achocolatada & 24 & 8,3 \\
Refrigerante garrafa & 11 & 3,7 \\
Suco de copo & 11 & 3,7 \\
Suco de caixinha & 10 & 3,4 \\
Água com gás & 8 & 2,7 \\
Isotônico & 5 & 1,7 \\
Energético & 3 & 1,0 \\
Chá & 3 & 1,0 \\
Refrigerante lata zero & 1 & 0,4 \\
Total & 294 & 100,0 \\
\hline Fonte: Dos autores, 2016. & &
\end{tabular}




\section{DISCUS5ÃO}

Existe grande preocupação com a quantidade e qualidade de alimentos consumidos, ${ }^{5}$ especialmente em função de mudanças drásticas nos padrões alimentares com repercussões sobre doenças crônicas não transmissíveis. ${ }^{2-4,12}$ Métodos tradicionais como diários alimentares, R24H e QFAs, ${ }^{5,18-20}$ nem sempre são aplicáveis à análise de hábitos alimentares compartilhados por grupos de indivíduos em um mesmo espaço.

Constatou-se, neste estudo, maior quantidade de resíduos de alimentos em relação às bebidas. Houve expressiva quantidade de alimentos e bebidas classificados como ultraprocessados. ${ }^{2}$ Por serem hipercalóricos e pouco nutritivos ${ }^{8}$ interferem negativamente na saciedade ${ }^{2}$ e, consequentemente, sobre a saúde.

Verificou-se que a maior parte dos alimentos e bebidas consumidos possuíam açúcares livres na sua composição. Tal fato é bastante preocupante, uma vez que estes competem com a ingestão de alimentos mais adequados sob o ponto de vista nutricional e associam-se a doenças crônicas como cárie dentária e obesidade, ${ }^{13}$ podendo, inclusive, estar relacionados à dependência química. ${ }^{10,11,16,17}$

A presença de bebedouros com copos descartáveis próximos às salas de aula pode ter sido um fator para elucidar a menor quantidade de bebidas consumidas. Por outro lado, a presença massiva de alimentos ultraprocessados pode ser explicada por fatores como a facilidade de armazenamento, transporte e preço dos alimentos industrializados, podendo ser adquiridos independentemente da oferta na única cantina da instituição. Além disso, pelo fato de poderem ser consumidos durante as aulas, uma vez que, em sua maioria, são inodoros e não exigem utensílios específicos como pratos, talheres e outros.

Há mais de 10 anos, a Portaria Interministerial $\mathrm{N}^{\circ}$. 1010/2006, ${ }^{3}$ traz diretrizes para a alimentação saudável em escolas do Brasil. Mesmo assim, há carência de normatizações para a alimentação em instituições de ensino superior.

Identificou-se a necessidade de serem instituídas medidas para melhorar os hábitos alimentares de universitários, especialmente em se tratando de futuros profissionais da saúde, uma vez que os exemplos influenciam práticas.

Mesmo que a temática da alimentação saudável e dos malefícios dos açúcares seja recorrente nas disciplinas oferecidas ao longo da formação de estudantes de Odontologia, evidenciou-se que a disponibilização de informações, por si só, é insuficiente para a mudança dos hábitos alimentares.

A recente diretriz “Ingestão de açúcares por adultos e crianças"13 deve ser amplamente discutida na formação dos profissionais da saúde, uma vez que recomenda a baixa ingestão de açúcares livres ao longo de toda a vida e a redução da ingestão de açúcares livres a menos de $10 \%$ da ingestão calórica total para crianças e adultos.

É preciso restringir a oferta e a venda de alimentos com alto teor de gordura, açúcar livre e sal e aumentar a oferta e promover o consumo de opções saudáveis, como as frutas. ${ }^{2}$ Além disso, é preciso estimular serviços de alimentação com opções saudáveis e, também, auxi- liar os indivíduos a escolherem os alimentos saudáveis. ${ }^{3}$ Medidas de promoção da saúde precisam ser instituídas com vistas ao maior controle dos indivíduos e grupos sobre os determinantes da saúde.

Embora a forma de coleta dos dados apresentados neste estudo apresente como limitações a impossibilidade de identificar quem consumiu os itens descartados e a presença de embalagens não significar, necessariamente, a totalidade consumida (possibilidade de sub ou superestimação), identificou-se que ela trouxe importantes ganhos em relação aos métodos tradicionais, como a não interferência nas preferências alimentares e o fato de não precisar de abordagens individualizadas, as quais exigem grande nível de tempo e cooperação, como é o caso de entrevistas e aplicação de questionários. Acredita-se que o método utilizado possa ser aplicado para análise de alimentos e bebidas consumidos por grupos de indivíduos em espaços diversos de uso comum, como é o caso das salas de aula, após atividades em grupo e em eventos, servindo de subsídios para ações educativas em saúde direcionadas a públicos específicos.

Considerando a importância da alimentação para a saúde, ${ }^{2-4}$ entende-se que a verificação dos hábitos alimentares, com vistas à elaboração de orientações e atividades de educação em saúde, deve ser integrante das ações de diferentes profissionais da saúde.

\section{CONSIDERACÕ̃ES FINAIS}

A análise dos alimentos e bebidas consumidos por estudantes de Odontologia, através dos resíduos presentes em lixeiras, evidenciou a predominância de hábitos alimentares não saudáveis durante a permanência dos estudantes nas salas de aula.

O padrão alimentar observado configura-se como fator de risco para doenças crônicas. Em função disso, estimula-se a construção de estratégias para promover hábitos alimentares positivos entre estudantes, como a realização de atividades de educação em saúde e o incentivo à oferta e à comercialização de alimentos e bebidas saudáveis na instituição de ensino pesquisada.

Entende-se, por fim, que o uso de métodos alternativos para a verificação do consumo de alimentos e bebidas por grupos de indivíduos, como o banner e a ficha "Quadro de alimentos consumidos", utilizados neste estudo, possam ser adaptados e aplicados em novas investigações e abordagens.

\section{REFERÊNCIAS}

1. World Health Organization (WHO). Health promotion, 2016. Disponível em: <http://www.who.int/healthpromotion/ conferences/previous/ottawa/en/\#>. Acesso em: 11 maio 2016.

2. Ministério da Saúde (MS). Guia Alimentar da População Brasileira, 2014. Disponível em: http://bvsms.saude. gov.br/bvs/publicacoes/guia _ alimentar _ populacao _ brasileira_2ed.pdf Acesso em 15 maio 2016.

3. Ministério da Saúde (MS). Portaria Interministerial $\mathrm{N}^{\circ}$. 1010/2006, 2006. Disponível em http://bvsms.saude.gov. $\mathrm{br} / \mathrm{bvs} /$ saudelegis/gm/2006/pri1010_ 08 _ 05 _ 2006.html/. Acesso em: 15 maio 2016 
4. World Health Organization (WHO). Diet, nutrition and the prevention of chronic diseases: report of a joint WHO/FAO expert consultation, 2003. Disponível em: http://apps.who. int/iris/bitstream/10665/42665/1/WHO_TRS_916.pdf. Acesso em: 07 jul. 2016

5. Freire MCM. Dieta, saúde bucal e saúde geral. In: Buischi YP. Promoção de saúde bucal na clínica odontológica. São Paulo (SP): Artes Médicas \& EAPAPCD. 2000. p. 248-78.

6. Associação Brasileira de Colite Ulcerativa e Doença de Crohn (ABCD). Sobre a Doença de Crohn, 2016. Disponível em: http:// abcd.org.br/sobre-a-doenca-de-crohn/. Acesso em: 16 maio 2016.

7. Freire MCM, Cannon G; Sheiham A. Análise das recomendações internacionais sobre o consumo de açúcares publicadas entre 1961 e 1991. Revista de Saúde Pública 1994;28(3):228-37. doi: 10.1590/S0034 89101994000300011

8. Organização Mundial de Saúde (OMS). Estratégia global em alimentação saudável, atividade física e saúde, 2004. Disponível em: http://189.28.128.100/nutricao/docs/geral/ ebPortugues.pdf. Acesso em: 23 maio 2016.

9. Pinto VG. Açúcares: suas relações epidemiológicas e econômicas com a cárie dental. In: Pinto VG. Saúde Bucal Coletiva. São Paulo (SP): Editora Santos. 2008. p. 479-508.

10. Rosa MA. Abuso e dependência de açúcares extrínsecos não lácticos: desenvolvimento de um instrumento diagnóstico e verificação de dependência de uma amostra de obesos e não obesos da cidade de Porto Alegre [tese]. [Porto Alegre (RS)]: Universidade Federal do Rio Grande do Sul; 2005. 183 p.

11. Rosa MAC. Dependência de açúcares: investigação dos critérios de dependência do DSM-IV adaptados para açúcar de adição [tese]. [Belo Horizonte (MG)]: Universidade Federal de Minas Gerais; 2011. 165 p.
12. Slavutzky SMB. Reflexões sobre a cárie dentária e outras doenças crônico-degenerativas. Ação Coletiva 1998;1(3):12-4.

13. World Health Organization (WHO). Guideline: sugars intake for adults and children, 2015. Disponível em: http://apps. who.int/iris/bitstream/10665/149782/1/9789241549028 eng.pdf?ua $=1$. Acesso em: 25 maio 2016.

14. Maltz M, Silva BB. Cárie dental: fatores relacionados. In: Pinto VG. Saúde Bucal Coletiva. São Paulo (SP): Editora Santos. 2008. p. 385-407.

15. Scientific Advisory Committee on Nutrition (SACN). Carbohydrates and health. London: TSO. 2015. 384 p.

16. Malheiros LS. Cárie e dependência de açúcares extrínsecos não lácticos [dissertação]. [Porto Alegre (RS)]: Universidade Federal do Rio Grande do Sul; 2007. 76 p.

17. Rosa MAC, Slavutzky SMB, Pechansky F, Kessler F. Processo de desenvolvimento de um questionário para avaliação de abuso e dependência de açúcar. Cadernos de Saúde Pública 2008;8(24):1869-76. doi: 10.1590/S0102$311 \times 2008000800015$.

18. Bezerra $A C B$, Toledo $O A$. Nutrição, dieta e cárie. In: Kriger L. ABOPREV: promoção de Saúde Bucal. São Paulo (SP): Editora Artes Médicas, 1999. p. 2-26.

19. Universidade de São Paulo (USP). Questionário de Frequência Alimentar Adultos, 2013. Disponível em: http://www.gacusp.com.br/resources/QFA\%20-\%202013.pdf. Acesso em: 15 maio 2016.

20. 20. Gonçalves FA, Pechansky F, Slavutzky SMB. Desenvolvimento de um Questionário de Frequência Alimentar (QFA-açúcar) para quantificar o consumo de sacarose. Revista HCPA 2011;31(4):428-36.

21. Pereira MG. Epidemiologia: teoria e prática. Rio de Janeiro: Editora Guanabara Koogan. 1999. 596 p.

Como citar: LUCIETTO, Deison Alencar et al. Alimentos e bebidas consumidos por estudantes de odontologia: subsídios para a construção de estratégias de promoção à alimentação saudável. Cinergis, Santa Cruz do Sul, v. 17, n. 3, set. 2016. ISSN 2177-4005. Disponível em: <https://online.unisc.br/seer/index.php/cinergis/article/view/7881 >. Acesso em: 30 set. 2016. doi:http://dx.doi.org/10.17058/cinergis.v17i3.7881. 
Sala:

Data:

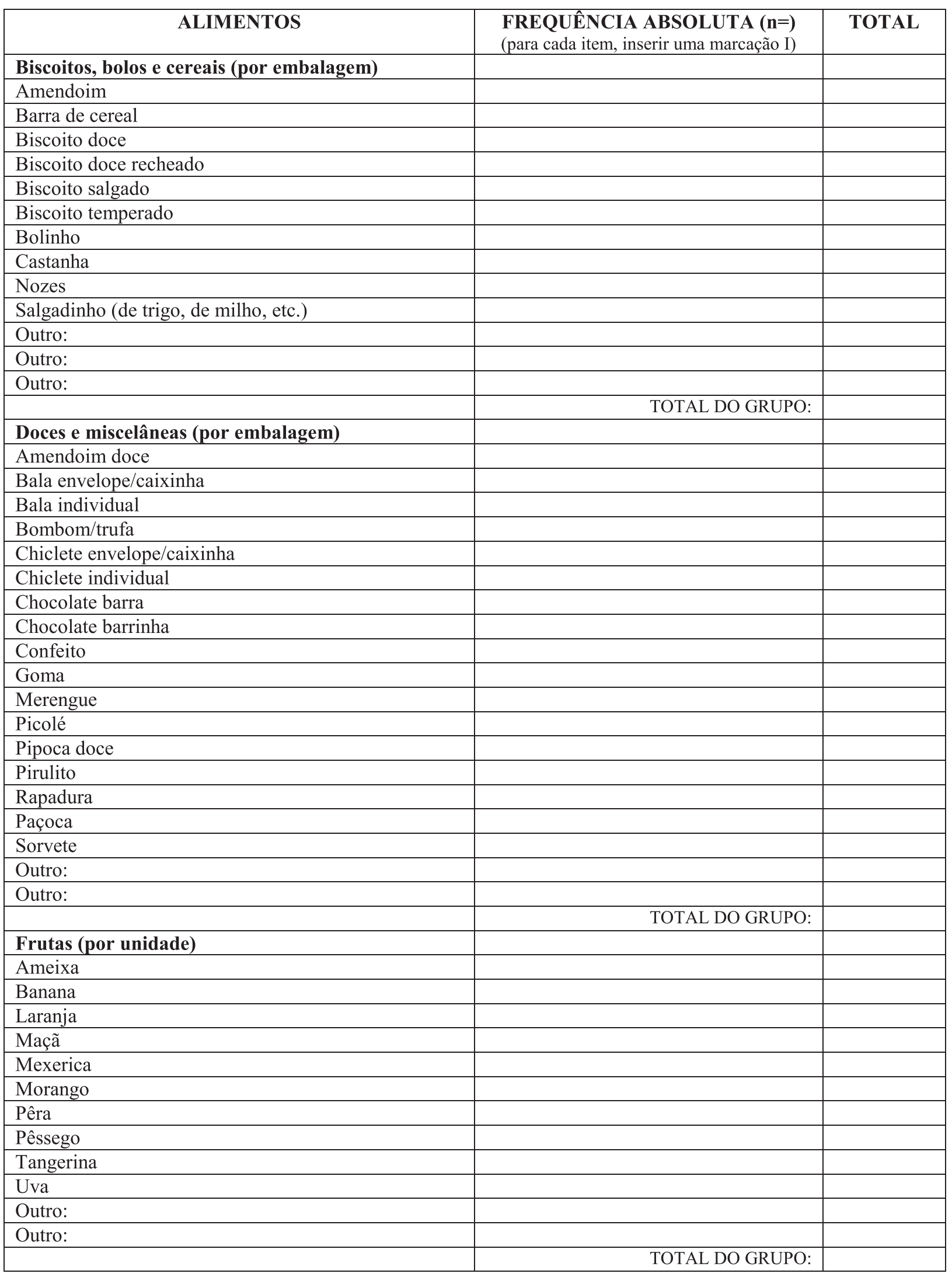




\section{QUADRO DE ANÁLISE DOS ALIMENTOS CONSUMIDOS}

\begin{tabular}{|c|c|c|}
\hline ALIMENTOS & & TOTAL \\
\hline \multicolumn{3}{|l|}{ Iogurtes (por embalagem) } \\
\hline \multicolumn{3}{|l|}{ Iogurte desnatado } \\
\hline \multicolumn{3}{|l|}{ Iogurte de frutas } \\
\hline \multicolumn{3}{|l|}{ Iogurte light } \\
\hline \multicolumn{3}{|l|}{ Iogurte natural } \\
\hline \multicolumn{3}{|l|}{ Outro: } \\
\hline \multicolumn{3}{|l|}{ Outro: } \\
\hline \multicolumn{3}{|l|}{ Outro: } \\
\hline & TOTAL DO GRUPO: & \\
\hline BEBIDAS & $\begin{array}{l}\text { FREQUÊNCIA ABSOLUTA }(\mathbf{n}=) \\
\text { (para cada item, inserir uma marcação I) }\end{array}$ & \\
\hline \multicolumn{3}{|l|}{ (por embalagem) } \\
\hline \multicolumn{3}{|l|}{ Água com gás } \\
\hline \multicolumn{3}{|l|}{ Água sem gás } \\
\hline \multicolumn{3}{|l|}{ Bebida achocolatada } \\
\hline \multicolumn{3}{|l|}{ Copo de café } \\
\hline \multicolumn{3}{|l|}{ Energético } \\
\hline \multicolumn{3}{|l|}{ Isotônico } \\
\hline \multicolumn{3}{|l|}{ Refrigerante garrafa } \\
\hline \multirow{2}{*}{\multicolumn{3}{|c|}{ Refrigerante garrafa zero }} \\
\hline & & \\
\hline \multicolumn{3}{|l|}{ Refrigerante lata zero } \\
\hline \multicolumn{3}{|l|}{ Suco de caixinha } \\
\hline \multicolumn{3}{|l|}{ Suco de copo } \\
\hline \multicolumn{3}{|l|}{ Outro: } \\
\hline \multicolumn{3}{|l|}{ Outro: } \\
\hline & TOTAL DO GRUPO: & \\
\hline
\end{tabular}

\section{Observações:}

